\title{
Detection of oxidized methionine in selected proteins, cellular extracts and blood serums by novel anti-methionine sulfoxide antibodies
}

\author{
Derek B. Oien ${ }^{\mathrm{a}}$, Tamar Canello ${ }^{\mathrm{b}}$, Ruth Gabizon ${ }^{\mathrm{b}}$, Maria Gasset ${ }^{\mathrm{c}}$, Brandi L. Lundquist ${ }^{\mathrm{a}}$, Jeff M. Burns ${ }^{\mathrm{d}}$, \\ Jackob Moskovitz ${ }^{\mathrm{a}, *}$ \\ ${ }^{a}$ Department of Pharmacology and Toxicology, School of Pharmacy, University of Kansas, Lawrence, KS 66045, USA \\ ${ }^{\mathrm{b}}$ Department of Neurology, The Agnes Ginges Center for Human Neurogenetics, Hadassah University Hospital, 91120 Jerusalem, Israel \\ 'Instituto Química-Física "Rocasolano", CSIC, 28006 Madrid, Spain \\ d Department of Neurology, KU Medical Center, University of Kansas, KS, USA
}

\section{A R T I C L E I N F O}

\section{Article history:}

Received 26 November 2008

and in revised form 17 January 2009

Available online 25 January 2009

\section{Keywords:}

Oxidative stress

Post-translation modification

Aging

Neurodegenerative diseases

Alzheimer's disease

Prion protein

Serum proteins

\begin{abstract}
A B S T R A C T
Methionine sulfoxide (MetO) is a common posttranslational modification to proteins occurring in vivo. These modifications are prevalent when reactive oxygen species levels are increased. To enable the detection of MetO in pure and extracted proteins from various sources, we have developed novel antibodies that can recognize MetO-proteins. These antibodies are polyclonal antibodies raised against an oxidized methionine-rich zein protein (MetO-DZS18) that are shown to recognize methionine oxidation in pure proteins and mouse and yeast extracts. Furthermore, mouse serum albumin and immunoglobulin (IgG) were shown to accumulate MetO as function of age especially in serums of methionine sulfoxide reductase A knockout mice. Interestingly, high levels of methionine-oxidized IgG in serums of subjects diagnosed with Alzheimer's disease were detected by western blot analysis using these antibodies. It is suggested that anti-MetO-DZS18 antibodies can be applied in the identification of proteins that undergo methionine oxidation under oxidative stress, aging, or disease state conditions.
\end{abstract}

(C) 2009 Elsevier Inc. All rights reserved.

\section{Introduction}

Oxidative damage to proteins is considered to be one of the major causes of aging and age-related diseases, and thus mechanisms have evolved to prevent or reverse these modifications. Methionine $(\mathrm{Met})^{1}$ oxidation is one of the most common posttranslational modifications to proteins mediated by reactive oxygen species (ROS) that may alter protein structure and function. During conditions of oxidative stress, there is an accumulation of proteins containing methionine sulfoxide (MetO) [1,2]. Insufficient reversal of protein-MetO to Met by the methionine sulfoxide reductase system (Msr family consisting of MsrA and MsrB) could cause certain proteins to loose their function, aggregate, and be toxic to the cell [1-4]. This function of the Msr system is supported by the fact that Met oxidation can denature proteins and convert the hydrophobic properties of Met into hydrophilic properties; thereby causing structural alterations to selected proteins that may affect their function and may be reversed by the Msr system in vivo [5]. Thus

\footnotetext{
* Corresponding author. Fax: +1 7858643536.

E-mail address: moskovij@ku.edu (J. Moskovitz).

1 Abbreviations used: Met, methionine; MetO, methionine sulfoxide; ROS, reactive oxygen species; Cys, cysteine; AD, Alzheimer's disease; PBS, phosphate-buffered saline; GS, glutamine synthetase; GAPD, glyceraldehyde 3-phosphate dehydrogenase; PrP, prion protein; MSA, mouse serum albumin.
}

far, Met residue oxidation has been shown to affect the activities of many proteins, mostly in vitro [3]. Examples include the potassium channel of the brain (as demonstrated in an ex vivo system) [6], an isoform of the inhibitory protein $\kappa \mathrm{B}[7,8]$, and calmodulin [9]. The general role of MsrA as a protein-function regulator and as antioxidant has been demonstrated in various tissues. For example, a recent report has emphasized the importance of MsrA in the reversal of calcium/calmodulin-dependent protein kinase II oxidation [10]. These findings suggest a key role for MsrA in controlling MetO reduction in proteins as well as the expression of genes involved in cellular antioxidant defense mechanisms. Identifying MetO-harboring proteins will greatly enhance the knowledge about processes leading to cellular malfunction associated with protein damage; thereby providing important information for the development of novel therapeutics against oxidative stress-associated diseases.

The endosperm of maize (Zea mays) seeds contains several classes of alcohol-soluble storage proteins called zeins, which together make up nearly $50 \%$ of the total seed protein content. Some zeins proteins are rich in the sulfur amino acids Met and cysteine (Cys). Therefore, these proteins could be very useful in designing a high rich-MetO protein. Zeins can be separated into four major classes (as determined by their sequence and apparent molecular mass following gel electrophoresis): $\alpha$ (19 and $22 \mathrm{kDa}), \beta$ (15 kDa), $\gamma(16$ and $27 \mathrm{kDa})$ and $\delta(10 \mathrm{kDa})$ zeins. In this study, 
we have used a derivative of the gene encoding an $18 \mathrm{kDa}$ Met-rich zein that is closely related to the $10 \mathrm{kDa}$ zein proteins [11]. The zein proteins, $10 \mathrm{kDa}$ (DZS10) and the $18 \mathrm{kDa}$ (DZS18), share $80 \%$ percent similarity and 66\% identity while the DZS18 contains $25 \%$ of Met residues compared with $23 \%$ of DZS10 [11]. The high percentage of Met residues in DZS18 (while having no Cys residues) makes it the most Met-rich protein among cereals and legumes [11]. Both $\delta$-zein genes are coordinately and temporally up regulated during endosperm development. It is suggested that their corresponding proteins may be a good source for free Met supply needed during the plant's later stages of development. Consequently, the zein proteins can serve as Met-rich substrate for oxidation resulting in MetO-rich proteins.

In the current study, we apply novel antibodies that can identify MetO in proteins (using MetO-zein as an antigen; patent pending) to probe for the presence of MetO in various pure proteins and in biological extracts. The newly developed antibodies will facilitate the determination and identification of proteins with increased levels of MetO from the aging process, conditions of oxidative stress, and disease state. Accordingly, we have used cellular protein extracts from organisms lacking the MsrA protein ( $m s r A$ null mutant yeast and $\mathrm{MrAA}^{-} /^{-}$mice) and their wild type controls, oxidized and scrapie prion protein, blood serum from young and aged mouse strains, and blood serum of healthy and patients diagnosed with Alzheimer's disease (AD).

\section{Materials and methods}

\section{Overexpression and purification of the recombinant DZS18 protein}

The DZS18 open reading frame was amplified by PCR reaction using 5' forward primer harboring BamHI restriction site and 3' reverse complementary primer harboring HindIII restriction site. The PCR reaction mixture contained the $5^{\prime}$-forward DNA primer starting at the $5^{\prime}$ end of the DZS18 open reading frame (66 bp downstream of the first ATG codon); the $3^{\prime}$-reverse complement DNA primer starting at the untranslated region forwarding towards the DZS18 stop codon, and cDNA for Zea mays (BioChain, Hayward, CA). The resulting PCR product was digested with BamHI and HindIII enzymes and subcloned into a pQE30 vector (Qiagen) at the complementary restriction sites. This created an open reading frame for a fusion protein between the $6 \times$ His tag and the zein $\mathrm{N}$ terminus, starting at the DZS18-DNA sequence corresponding to the 22 nd amino acid of the protein. The pQE30 $6 \times$ His tag was inserted in frame with the N-terminus of the DZS18 and the construct was transformed and expressed in E. coli. Following overexpression of the fusion protein in bacteria, the recombinant protein was extracted from the bacterial cells and purified on an affinity nickel column, according to the manufacture procedure (BD Biosciences). Then, the protein was oxidized with $0.3 \%$ hydrogen peroxide for $2 \mathrm{~h}$ at $37^{\circ} \mathrm{C}$. The oxidized protein (MetO-DZS18) was dialyzed against phosphate-buffered saline (PBS) at $\mathrm{pH} 7.4$ to remove remaining oxidant and subjected to amino acid analysis, as previously described [2]. The protein was found to be fully oxidized in its Met moiety (by amino acid analysis [2]; data not shown) and consequently injected into a rabbit over several time intervals to produce anti-MetO-DZS18 antibodies.

\section{Purified proteins serving as substrates for the anti-MetO-DZS18} antibodies

Glutamine synthetase and glyceraldehyde 3-phosphate dehydrogenase

Purified recombinant glutamine synthetase (GS; a gift from Mrs.

Berlett, NHLBI) and glyceraldehyde 3-phosphate dehydrogenase (GAPD purchased from Sigma) were incubated in the presence and absence of $0.3 \%$ of $\mathrm{H}_{2} \mathrm{O}_{2}$ for $2 \mathrm{~h}$ at $37^{\circ} \mathrm{C}$. Thereafter, equal amounts of these proteins were subjected to SDS-gel electrophoresis followed by western blot analysis using the anti-MetO-DZS18 antibodies.

\section{Prion protein}

Full length polypeptide chains corresponding to the hamster prion (PrP) sequence, lacking the $\mathrm{N}$ - and $\mathrm{C}$-terminal sequences were expressed from their pET11a construct and folded to their $\alpha$-form as previously described [12]. The enriched recombinant PrP protein, rSHaPrP (Residues 23-230), was then oxidized in the presence and absence of $50 \mathrm{mM} \mathrm{H}_{2} \mathrm{O}_{2}$ for $30 \mathrm{~min}$ at room temperature. For the purified Proteinase $\mathrm{K}$ resistant $\operatorname{PrP}^{\mathrm{Sc}}$ samples, membranes from scrapie infected hamster brain homogenates were subjected to extraction by sarkosyl followed by ultracentrifugation, gradient enrichment, and PK digestion as previously described $[13,14]$. Thereafter, equal amounts of these proteins were subjected to SDS-gel electrophoresis followed by western blot analysis using either the anti-MetO-DZS18 antibodies, or $\alpha$-PrP mAb IPCV1 (monoclonal antibodies against PrP) [15], or $\alpha$-PrP mAb IPC2 (monoclonal antibodies which recognizes PrP with intact disulfide bond) [15].

\section{Detection of MetO-containing proteins in tissue/cell extracts}

\section{Mouse tissues}

To further characterize the anti-MetO-DZS18 antibodies for detection of MetO-containing proteins in biological extracts, protein extracts from several mouse tissues were treated with and without $100 \mathrm{mM} \mathrm{H}_{2} \mathrm{O}_{2}$ for $2 \mathrm{~h}$ at $37{ }^{\circ} \mathrm{C}$ in the presence of $2 \%$ SDS (to inhibit peroxidases activities). Thereafter, equal amounts of protein extracts were subjected to SDS-gel electrophoresis followed by western blot analysis using the anti-MetO-DZS18 antibodies.

\section{Yeast cells}

To determine whether the anti-MetO-DZS18 antibodies are capable of detecting oxidized proteins in yeast, two yeast strains were grown in the presence or absence of $\mathrm{H}_{2} \mathrm{O}_{2}$. One strain was enriched in its MetO-reduction ability (OP, an overproducing strain of MsrA) and one strain was compromised in its MetO-reduction ability (MT, a null mutant strain of $m s r A$ ). The yeast strains OP and MT were grown in the presence or absence of $1 \mathrm{mM} \mathrm{H}_{2} \mathrm{O}_{2}$ till their growth rate reached 150 klett units. Following their growth, the cells were harvested, extensively washed with PBS and disrupted in the presence of PBS and proteases inhibitors cocktail (Roche). Equal amounts of protein extracts from each strain were subjected to an SDS-gel electrophoresis followed by western blot analysis using the anti-MetO-DZS18 antibodies. To compete for the binding of the antibodies to the targeted proteins, the antibodies were incubated in the presence of $100 \mathrm{mM}$ free L-MetO in a duplicate experiment (in parallel, L-MetO was substituted with L-Met to serve as a control experiment to the L-MetO binding competition to the antibodies).

\section{Blood serum proteins}

Aliquots of blood samples (100-200 $\mu \mathrm{L})$ were isolated from wild type and $\mathrm{MrAA}^{-} /^{-}$mouse strains at ages 6, 12 and 16 months. Following serum extraction, equal amounts of serum protein from each sample (measured by the Bradford reagent (Bio-Rad)) were subjected to SDS-gel electrophoresis and western blot analysis using the anti-MetO-DZS18 antibodies.

Similarly, human serums from healthy and Alzheimer's patients donors were analyzed as well (five individuals from each group with matching ages between $70-80$ years old, provided by Dr. Jeff 
Burns clinic KUMC, University of Kansas). Additionally, to determine MetO content in mouse serum albumin the protein was purified by separating mouse serum in SDS-gel electrophoresis and extracting the corresponding band, digesting it with trypsin, and subjecting the resulting peptides to mass spectrometry analysis. A parallel gel was ran and analyzed for the presence of MetO in the serum albumin by western blot analysis using the anti-MetODZS18 antibodies.

\section{In-gel digestion for mass spectrometry analysis}

The mouse serum albumin band was excised from the gel and washed with $0.2 \mathrm{M}$ ammonium bicarbonate/50\% acetylnitrile at $37^{\circ} \mathrm{C}$ for $45 \mathrm{~min}$ with gentle agitation. After discarding the washing solution, the gel was dried in a CentiVap Concentrator (Labconco) and incubated with $150 \mu \mathrm{l}$ of $0.2 \mathrm{M}$ ammonium bicarbonate and $20 \mu \mathrm{l}$ of $100 \mathrm{mM}$ DTT solution at $60^{\circ} \mathrm{C}$ for $30 \mathrm{~min}$. Then, $40 \mu \mathrm{l}$ of $100 \mathrm{mM}$ iodoacetamide was added and the sample was incubated at room temperature for $30 \mathrm{~min}$. After discarding the solution the gel was washed with $200 \mu \mathrm{l}$ of $0.2 \mathrm{M}$ ammonium prior to the incubation with $20 \mu \mathrm{l}$ of the digestion buffer $\left(0.2 \mathrm{M}\right.$ ammonium bicarbonate $\left./ 5 \mathrm{mM} \mathrm{CaCl}_{2}\right)$ containing $0.3-$ $0.5 \mu \mathrm{g}$ trypsin at $37^{\circ} \mathrm{C}$ overnight. The resulting digested peptides were analyzed by mass spectrometry techniques for their amino acid sequences.

\section{Results}

Antibodies produced against Meto-DZS18 show specificity towards protein-MetO

The antibodies showed specificity towards the MetO-DZS18 protein and did not react with the non-oxidized form of the protein (DZS18). We have verified by using amino acid analysis for MetO detection [2] that indeed all Met residues in the MetO-DZS18 were oxidized while all other amino acid residues remained intact (data not shown). In addition, the antibodies reacted with several nonhomologues proteins in their oxidized forms: glutamine synthetase (GS) and glyceraldehyde 3-phosphate dehydrogenase (GAPD) (Fig. 1), suggesting specific reactivity of the antibodies towards MetO residues (all the Met residues in the oxidized proteins were found to be oxidized, as judged by amino acid analysis; data not shown). The MetO-18 (Fig. 1, 018) migrates as a $36 \mathrm{kDa}$ protein due to the mass contribution by the addition of $6 \times$ His tag to its $\mathrm{N}$-terminus and oxygen to all of its Met residues (this protein consists of $25 \%$ Met residues). The native GS shows reaction with antibodies (Fig. 1) as it is naturally oxidized under its recommended storage conditions at $4{ }^{\circ} \mathrm{C}$. However, the oxidized GS (OGS) reacted slightly stronger with the antibodies and migrated slower in the gel due its enhanced Met oxidation (Fig. 1). Moreover, the reaction intensity of the antibodies with the proteins seems to correlate with the percent of the Met residues in each tested protein $(018$, 25\%; OGS, 3.6\%; and OGAPD, 3.0\%), suggesting full Met oxidation in all proteins. To further characterize the anti-MetO-DZS18 antibodies for detection of MetO-containing proteins in biological extracts, protein extracts from several mouse tissues were treated with and without $100 \mathrm{mM} \mathrm{H} \mathrm{O}_{2}$ for $2 \mathrm{~h}$ at $37^{\circ} \mathrm{C}$ in the presence of $2 \% \mathrm{SDS}$ (to inhibit peroxidases activities). As expected, following western blot analysis using the anti-MetO-DZS18 antibodies, the reaction of the antibodies with existing and new protein bands was increased in all tissues that were treated with $\mathrm{H}_{2} \mathrm{O}_{2}$ in comparison to the non-treated proteins (Fig. 2). This result indicates that the anti-MetO-DZS18 antibodies are capable of detecting oxidized proteins in crude extracts due to MetO modification mediated by $\mathrm{H}_{2} \mathrm{O}_{2}$. The antigen MetO-DZS18 protein does not have any Cys

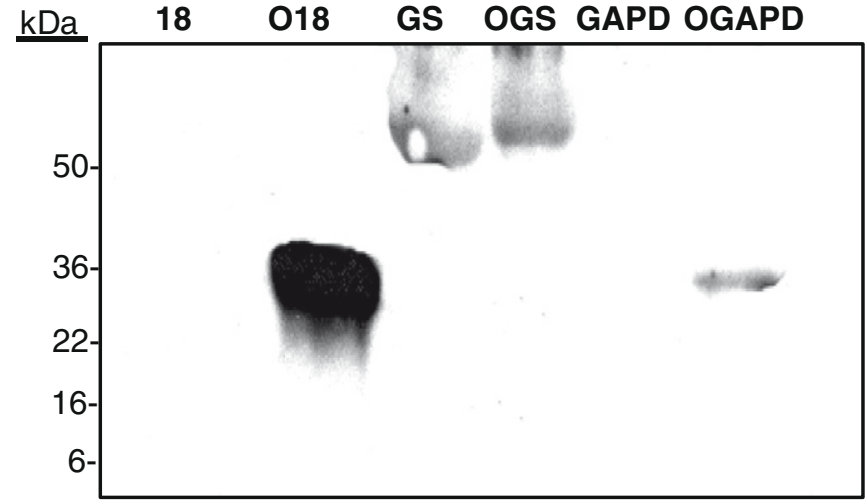

Fig. 1. The anti-MetO-DZS18 antibodies detect methionine sulfoxide in purified proteins. Western blot analysis of purified recombinant glutamine synthetase (GS) and glyceraldehyde 3-phosphate dehydrogenase (GAPD) were incubated in the presence and absence of $0.3 \%$ of $\mathrm{H}_{2} \mathrm{O}_{2}$ for $2 \mathrm{~h}$ at $37^{\circ} \mathrm{C}$. Equal amounts of theses proteins were subjected to SDS-gel electrophoresis followed by western blot analysis using the anti-MetO-DZS18 antibodies. OGS, oxidized GS; OGAPD, oxidized GAPD; 18, DZS18; 018, oxidized DZS18. kDa, molecular mass indicators (in kDa). The presented data represents three independent experiments.

nor other amino acids that are readily oxidized except Met. This protein did not show any actual amino acid oxidation except for Met (as determined by amino acid analysis (data not shown)); thereby making the MetO residues as the only possible binding target for the antibodies raised against MetO-DZS18. To determine whether the anti-MetO-DZS18 antibodies are capable of detecting oxidized proteins in vivo, the two yeast strains were exposed to $\mathrm{H}_{2} \mathrm{O}_{2}$. One strain was enriched in its MetO-reduction ability (OP, an overproducing strain of MsrA) and a one strain was compromised its MetO-reduction ability (MT, a null mutant strain of $m s r A)$. As expected, the MT strain that was more sensitive to Met oxidation produced a dominant protein band following reaction with the anti-MetO-DZS18 antibodies even without the $\mathrm{H}_{2} \mathrm{O}_{2}$ treatment. Moreover, the reaction with this protein band was enhanced

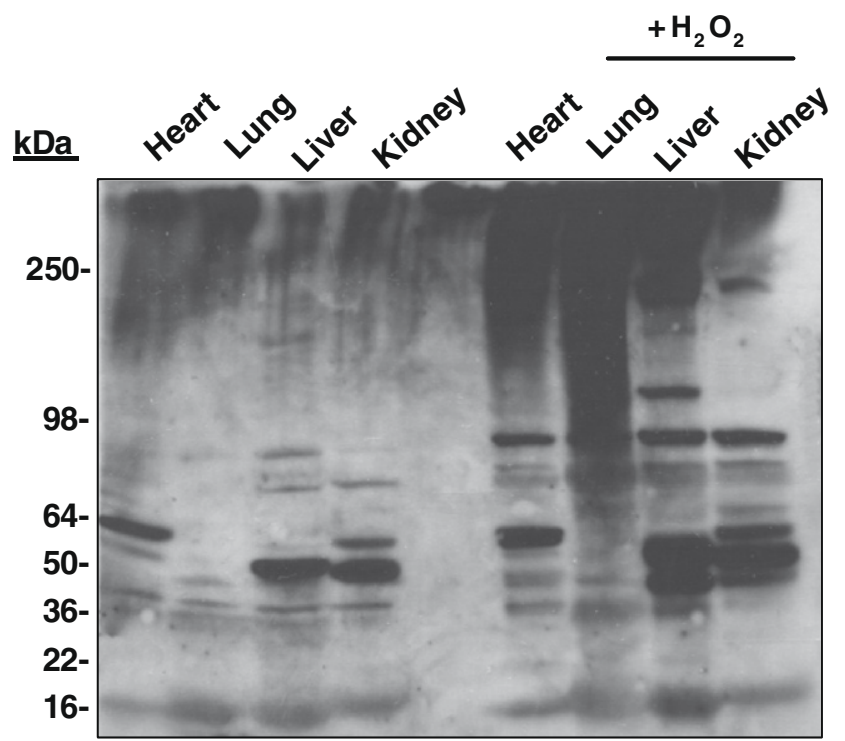

Fig. 2. The anti-MetO-DZS18 antibodies detect methionine sulfoxide in protein extracts of various mouse tissues. Protein extracts from several mouse tissues were treated with and without $100 \mathrm{mM} \mathrm{H}_{2} \mathrm{O}_{2}$ for $2 \mathrm{~h}$ at $37{ }^{\circ} \mathrm{C}$ in the presence of $2 \% \mathrm{SDS}$ (to inhibit peroxidases activities). Thereafter, equal amounts of protein extracts were subjected to SDS-gel electrophoresis followed by western blot analysis using the anti-MetO-DZS18 antibodies. kDa, molecular mass indicators (in kDa). The presented data represents three independent experiments. 
following exposure of the cells to $\mathrm{H}_{2} \mathrm{O}_{2}$ (Fig. 3). Accordingly, the OP strain to that is resistant to Met oxidation showed no detectable reactions with the anti-MetO-DZS18 antibodies (Fig. 3). The specificity of the antibodies to single MetO residues was supported by the fact that when free MetO was co-incubated with the antiMetO-DZS18 antibodies (during the western blot analysis) the reactivity of the protein band with the antibodies was diminished (Fig. 3); suggesting a competition between the free amino acid and the protein MetO residues on the binding to the respected antibodies. The competition was not complete as the competitor is an amino acid rather than protein residue and the antibodies may have subclasses that can bind multiple MetO residues only. Moreover, an identical control experiment performed in the presence of free Met, instead of free MetO, did not show any change in the reaction of the antibodies with the targeted protein (relative to the reaction without any amino acid, data not shown). We believe that the additional presented data provide supportive evidence for the concept that indeed the anti-MetO-DZS18 antibodies are reacting specifically towards multiple and single MetO residues. It is important to note, that the average percent of total Met residues in a protein is only $\sim 2 \%$, while the percentage of the surface exposed Met is even lower as Met by its nature is a hydrophobic amino acid. Therefore, most of the Met residues are predicted to be buried within the molecular structure of a protein, thereby becoming less excessive to oxidation by most oxidants).

\section{Detection of MetO in prion protein}

The prion type $\operatorname{PrP}^{\mathrm{Sc}}$ is the modified form of the membranal prion protein $\left(\mathrm{PrP}^{\mathrm{C}}\right)$. $\mathrm{PrP}^{\mathrm{Sc}}$ is considered to be a version of prion that is associated with transmissible spongiform encephalopathies. Stahl et al. have shown that either one or two Met residues (M206 and/or M213) were present in the form of MetO in $\mathrm{PrP}^{\mathrm{Sc}}$ [16]. Additionally, $\operatorname{PrP}^{\mathrm{C}}$ has been assigned a role in the protection of cells from ROS $[17,18]$ and recombinant $\operatorname{PrP}(\mathrm{rPrP})$ was a target for oxidation $[19,20]$. Recently, it has been demonstrated by Gabizon et al. that brain $\operatorname{PrP}^{\mathrm{Sc}}$ contains high levels of MetO, which are not detected in brain $\operatorname{PrP}^{C}$ and its recombinant models [15]. Accordingly, we have examined the possibility of detecting MetO in rPrP, oxi-

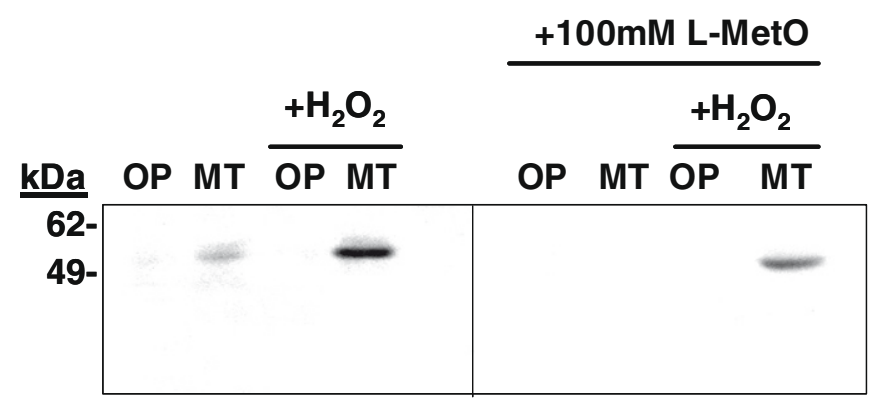

Fig. 3. The anti-MetO-DZS18 antibodies detect methionine sulfoxide in yeast protein extracts. Two yeast strains were grown in the presence or absence of $\mathrm{H}_{2} \mathrm{O}_{2}$. One strain was enriched in its MetO-reduction ability (OP, an overproducing strain of MsrA) and one strain was compromised in its MetO-reduction ability (MT, a null mutant strain of $m s r A$ ). The yeast strains: OP (MsrA overproducing strain) and MT (null mutant msrA strain) were grown in the presence or absence of $1 \mathrm{mM} \mathrm{H}_{2} \mathrm{O}_{2}$ till their growth rate reached 150 klett units. Following their growth, the cells were harvested, extensively washed with PBS, and disrupted in the presence of PBS and proteases inhibitors cocktail (Roche). Equal amounts of protein extracts from each strain were subjected to SDS-gel electrophoresis followed by western blot analysis using the anti-MetO-DZS18 antibodies. To compete for the antibodies' binding to the targeted proteins, the antibodies were incubated in the presence of $100 \mathrm{mM}$ free L-MetO in a duplicate experiment. L-Met did not show an ability to compete on the antibodies' binding (data not shown). kDa, molecular mass indicators (in kDa). The presented data represents three independent experiments. dized rPrP and Proteinase $\mathrm{K}(\mathrm{PK})$ resistant hamster $\operatorname{PrP}^{\mathrm{Sc}}$ by the anti-MetO-DZS18 antibodies. As shown in Fig. 4, the $\alpha$-PrP mAb IPCV1 antibodies recognized all forms tested in the figure; $\alpha$-PrP mAb IPC2 antibodies recognized PrP forms that were not oxidized at Met213 [15], and the anti-MetO-DZS18 antibodies recognized all forms of PrP tested, while exhibiting the strongest reaction against the oxidized form of rPrP. As seen in Fig. 4, while $\alpha$-PrP mAb IPC2 antibodies reacted with non-oxidized $\mathrm{rPrP}$ and with the lower forms of hamster $\operatorname{PrP}^{\mathrm{Sc}}$, the anti-MetO-DZS18 antibodies recognized all the additional $\operatorname{PrP}$ forms that react with $\alpha-\operatorname{PrP} \mathrm{mAb}$ IPCV1 antibodies. The fact that the silver stain pattern of $\operatorname{PrP}^{\mathrm{Sc}}$ is similar to the $\alpha$-PrP mAb IPCV1 pattern of PrP recognition suggests the anti-MetO-DZS18 antibodies indeed reacted with the fully glycosylated $\mathrm{PrP}^{\mathrm{Sc}}$ form not recognized by $\alpha$-PrP mAb IPC2 antibodies (primarily the $\sim 36 \mathrm{kDa}$ band that is mainly presented in the $\operatorname{PrP}^{\mathrm{Sc}}$ form). It is important to note that the gel electrophoresis performed prior to the western blot using $\alpha$-PrP mAb IPC2 antibodies was done in the absence of $\beta$-mercaptoethanol. This was due to the fact that $\alpha$-PrP mAb IPC2 antibodies can recognize only PrP proteins with intact disulfide bridges [15]. However, oxidation of $\operatorname{PrP}$ causing MetO formation interferes with the protein recognition by the $\alpha$-PrP mAb IPC2 antibodies (Fig. 4). Complementary to this observation, it is suggested that the presence of $\beta$-mercaptoethanol causes the MetO at position 213 to be more surface exposed to facilitating its recognition by the anti-MetO-DZS18 antibodies (Fig. 4). These results are consistent with the recent publication [15] indicating that several Met residues are oxidized in $\operatorname{PrP}^{\mathrm{Sc}}$. This suggests that the anti-MetO-DZS18 antibodies can be an important tool to study this modification in prion protein.

\section{Blood serum proteins}

The major proteins in serum, mouse serum albumin (MSA) as well of intact IgG, were shown to react in western blot analysis with the anti-MetO-DZS18 antibodies (Fig. 5). The identification of the MSA was confirmed by mass spectrometry analysis using trypsin-digested peptides covering about $25 \%$ of the protein sequence. Among all peptides analyzed, Met572 was shown to be oxidized to MetO. Apparently, Met572 is conserved in all sequenced serum albumins and perhaps its structural location promotes its availability to oxidation. The identification of an intact IgG as the other target for Met oxidation (Fig. 5) was confirmed by probing a parallel western blot with primary rabbit anti-mouse IgG (data not shown). Further western blot analyses for Met oxidation in MSA and IgG were performed on serums from wild type and $\mathrm{MsrA}^{-} \mathrm{J}^{-}$mice taken at various ages. As shown in Fig. 6A, the oxidation of MSA and IgG in its intact form ( $150 \mathrm{kDa})$ intensified in $\mathrm{MsrA}^{-} /^{-}$compared to wild type serums. The SDS-gel electrophoresis was performed in the presence of $\beta$-mercaptoethanol that is expected to reduce the disulfide bonds between each $\operatorname{IgG}$ monomer as well as between the heavy ( $\sim 50 \mathrm{kDa})$ and light $(\sim 25 \mathrm{kDa})$ chains of the protein. Accordingly, in serum of 6 -month-old mice the IgG molecules were reduced to their monomeric components and shown to contain MetO by western blot analysis (Fig. 6A). In contrast, with older age, the disulfide bond reduction was inhibited and the relative oxidative levels of intact IgG ( $\sim 150 \mathrm{kDa})$ and aggregated form of an unknown serum protein ( $250 \mathrm{kDa}$ suggested to be an aggregated form of $\operatorname{IgG}(\mathrm{Ag})$ were increased in both mouse strains as function of age (Fig. 6A). In comparison to wild type, the Met oxidation of the Ag form of $\operatorname{IgG}$ was mostly pronounced in $\mathrm{MsrA}^{-} \mathrm{I}^{-}$serums (Fig. 6A). Densitometry analysis for the intensities of the corresponding MSA, IgG and AG bands have confirmed that the MetO levels of the selected proteins increases in $\mathrm{MsrA}^{-} \mathrm{C}^{-}$versus wild type serums (Fig. 6B). Moreover, the levels of the MetO-Ag were increased in an age-dependent manner in both strains, while showing the highest levels in the $\mathrm{MsrA}^{-} /^{-}$strain 

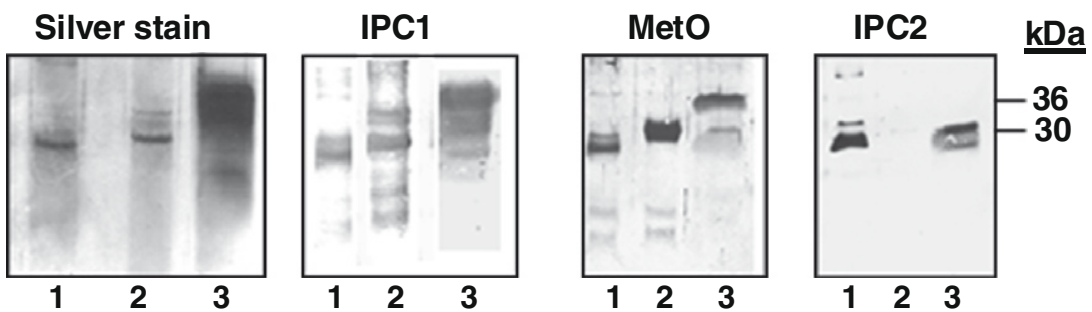

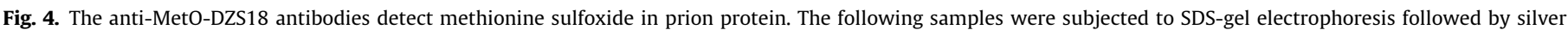

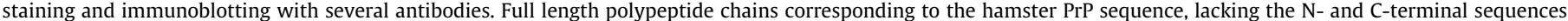

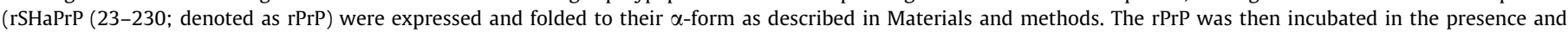

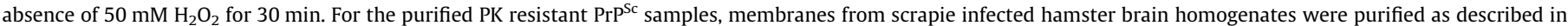

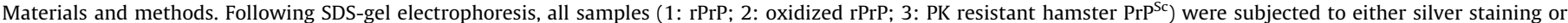

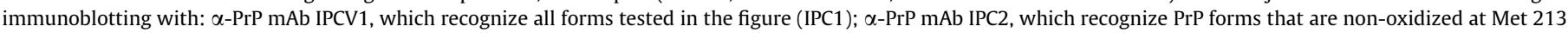

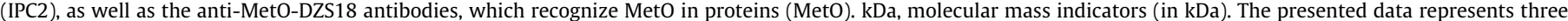
independent experiments.

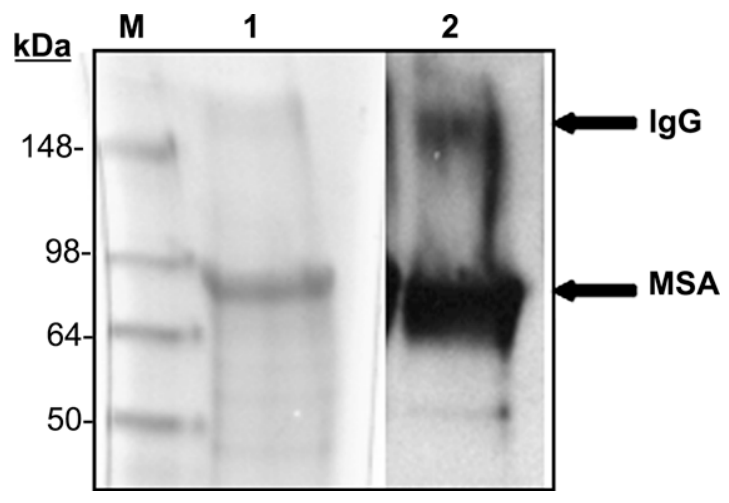

Fig. 5. The anti-MetO-DZS18 antibodies detect methionine sulfoxide in mouse serum proteins. Fifty microgram of mouse serum proteins were subjected to SDSgel electrophoresis and either stained with coomassie blue (1), or probed with antiMetO-DZS18 antibodies following western blot analysis (2). M, molecular mass markers (in $\mathrm{kDa}$ ). IgG-Immunoglobulin $\mathrm{G}$ that was detected in a parallel gel by primary goat anti-mouse IgG antibodies (GenWay; data not shown). MSA, mouse serum albumin (detected by mass spectrometry analysis according to procedures described in Materials and methods). The presented data represents three independent experiments.

(Fig. 6B). The latter observation may be due to the common nature of aggregated proteins of being more resistant to degradation, which fosters their accumulation with age. Similar to the observed effects of age and lack of MsrA on IgG MetO levels, intact forms of IgGs in serums of patients with AD were shown to contain MetO while none was observed in control serums, respectively (Fig. 7). It is noted that the limited number of human specimens analyzed for the presence of MetO in serum proteins requires further validation by larger group analyses.

\section{Discussion}

In the current study the development and characterization of novel anti-MetO antibodies is described. These anti-MetO-DZS18 antibodies were able to identify oxidized forms of purified proteins (Fig. 1) as well as oxidized proteins in various tissue extracts, respectively (Fig. 2). Likewise, the reaction of the antibodies with a major detectable protein in yeast extract intensified when the yeast strain lacked MsrA and was exposed to $\mathrm{H}_{2} \mathrm{O}_{2}$ (Fig. 3). In contrast, the reaction of the antibodies with the same protein diminished completely in protein extracts of an MsrA-overexpressing yeast strain and was reduced in the presence of free L-MetO. Thus, the presented data (Figs. 1-3) support the specificity of the anti-MetO-DZS18 antibodies toward protein-MetO. Very recently,
A
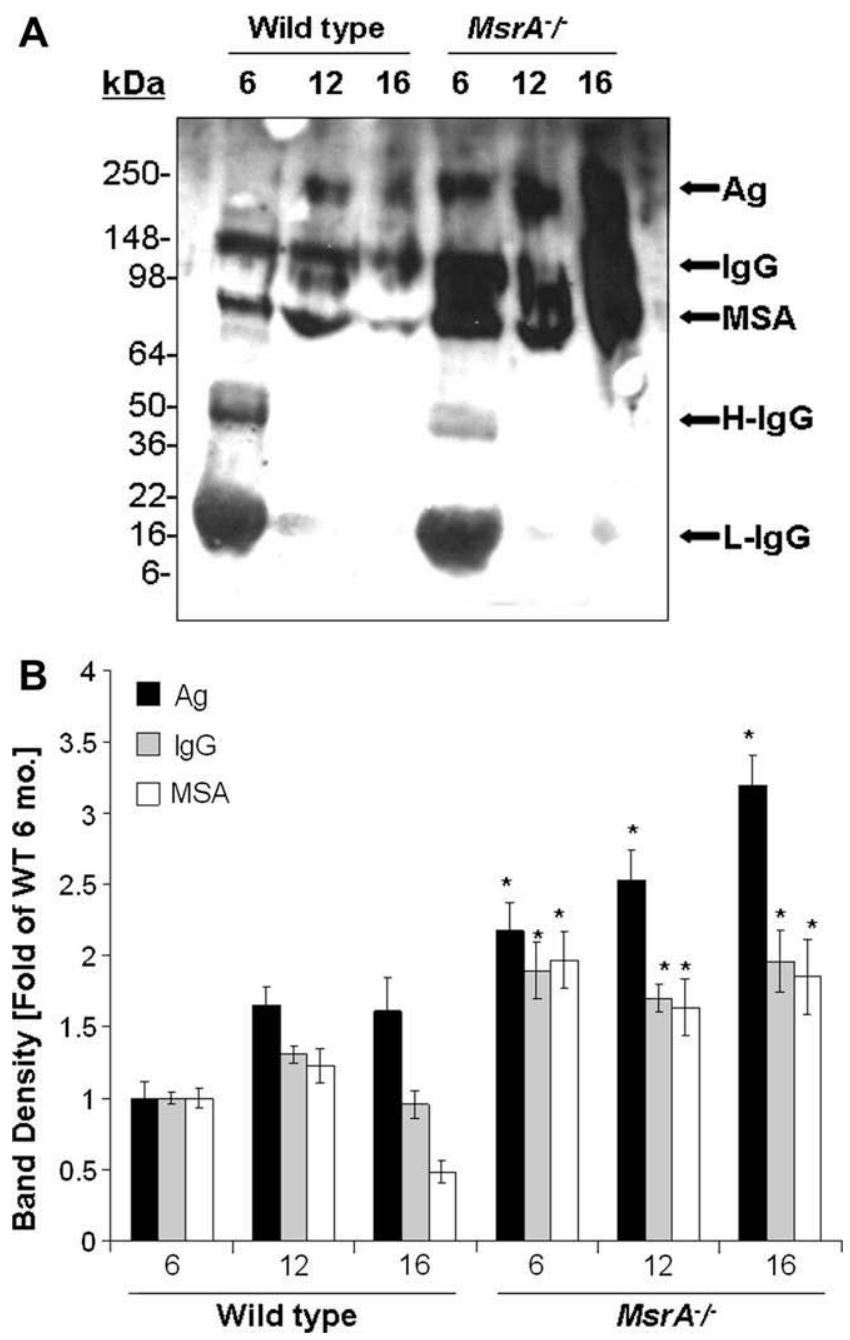

Fig. 6. The anti-MetO-DZS18 antibodies detect methionine sulfoxide in mouse serum proteins in an age-dependent manner. (A). Western blot analysis on mouse serums as function of age $(6,12$ and 16 , represent age in months) using the antiMetO-DZS18 antibodies. Ag, aggregated form of serum protein. IgG, native form of IgG. H-IgG, heavy chain of IgG. L-IgG, light chain of IgG. MSA, mouse serum albumin. $\mathrm{kDa}$, molecular mass indicators (in $\mathrm{kDa}$ ). (B). Densitometry analysis for the intensities of the MSA, IgG and Ag bands shown in panel A. The numbers 6, 12 and 16 represent age in months. The $\left({ }^{*}\right)$ symbol represents significant statistical difference $(P<0.05$; $t$-test) between the age-matching bands of both mouse strains. Also, there were significant statistical differences $(P<0.05 ; t$-test $)$ within the relative levels of the $\mathrm{Ag}$ bands in each mouse strain: between all age groups of $\mathrm{MrAA}^{-} \mathrm{I}^{-}$and between 6 and 12 or 16 months old of wild type mice .The shown data represent three independent experiments. 
Control

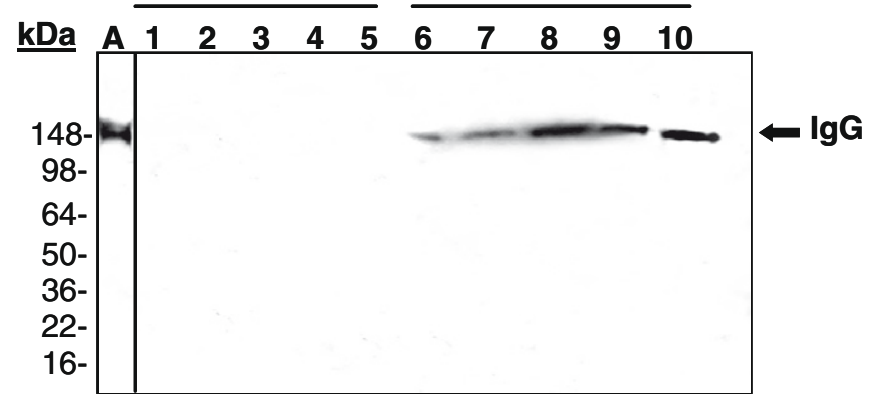

Fig. 7. The anti-MetO-DZS18 antibodies detect methionine sulfoxide in $\operatorname{IgG}$ in serum proteins of Alzheimer's disease human subjects. Western blot analysis on human serums using the anti-MetO-DZS18 antibodies (1-5, samples from control human subjects; 6-10, samples of AD patients; all samples are aged matched (7080 years old). A. A representative sample of human serum probed with primary rabbit anti-human IgG antibodies (GenWay). kDa, molecular mass indicators (in $\mathrm{kDa}$ ). The presented data represents three independent experiments.

similar antibodies were developed but were not characterized for their ability to recognize MetO in various proteins [21]. Methionine oxidation in proteins that are associated with in neurodegenerative diseases is thought to play a role in their toxicity and structure. For example, $\alpha$-synuclein in Parkinson's disease, $\beta$-amyloid in Alzheimer's disease and PrP in Creutzfeldt-Jakob disease (CJD) [4]. With respect to the latter protein, very limited literature is provided regarding the formation and consequence of MetO in PrP. One of the major problems in identifying MetO in $\mathrm{PrP}^{\mathrm{Sc}}$ is that it becomes proteinase $\mathrm{K}$ resistant and aggregates into structures that interfere with commonly used analytical processes for MetO determination (like mass spectrometry and amino acid composition analyses). Accordingly, the ability of the anti-MetO-DZS18 antibodies to recognize MetO in both oxidized $\mathrm{rPrP}$ and $\operatorname{PrP}^{\mathrm{Sc}}$ (Fig. 4) may serve as a tool to follow primary events that are causing the occurrence of high levels of oxidation and aggregation to $\mathrm{PrP}^{\mathrm{c}}$, which in turn are prompting the appearance of $\mathrm{PrP}^{\mathrm{Sc}}$. In the search of a marker for Met oxidation in proteins in vivo, we have examined the possibility of monitoring the levels of MetO in serum proteins, like serum albumin and immunoglobulin (IgG) that are the major proteins in serum. Indeed, serum albumin (Figs. 5 and 6) and IgG were shown to be prone to Met oxidation, especially in an antioxidantcompromised mice ( $\mathrm{MsrA}^{-} /{ }^{-}$; Fig. 6). The aggregated form of $\operatorname{IgG}$ (Ag) was also shown to exhibit age-dependent accumulations of MetO, with the highest levels observed in $\mathrm{MsrA}^{-} /^{-}$mouse (Fig. 6). It is suggested that with age or enhanced oxidative stress conditions (like in the case of $\mathrm{MrrA}^{-} /^{-}$), the levels of MetO in serum proteins are elevated and thus could serve as a marker to organism oxidative stress conditions and age (using the anti-MetO-DZS18 antibodies as a probe for MetO-proteins). For example, recently it was shown by mass spectrometry analyses that plasma albumin in patients treated with hemodialysis contained MetO [22]. Also, fully recombinant human monoclonal antibody was shown to contain MetO under chemical oxidation in vitro [23].

Neurodegenerative diseases are associated with increased conditions of oxidative stress leading to increase of protein oxidation. However, to best of our knowledge there is no reliable antibody that can show significant differences in serum protein oxidation between patients having $\mathrm{AD}$ and non-AD individuals. The use of the current novel antibodies showed that MetO in IgG is highly elevated in $\mathrm{AD}$ patients and thus may be applied in screening for the occurrence of $\mathrm{AD}$ following western blot analysis (Fig. 7). Further investigations with larger groups of AD patients and matched controls are under way to strengthen this observation. Nevertheless, in spite of the small number of tested samples, the striking relative high levels of MetO in serum IgG of individuals with AD suggest that the presence of MetO-IgG is associated with $\mathrm{AD}$ state and that although its levels are lower in human than in mouse serums they are still detectable by the anti-MetO-DZS18 antibodies. In summary, we present in this study novel anti-MetO antibodies that enable the identification of MetO-proteins, both as a mean to follow biological processes associated with oxidative stress and aging and as a screening tool for neurodegenerative diseases like AD.

\section{Acknowledgment}

This study was partially supported by the National Institute of Aging Grant AG027363.

\section{References}

[1] J. Moskovitz, B.S. Berlett, J.M. Poston, E.R. Stadtman, Proc. Natl. Acad. Sci. USA 94 (1997) 9585-9589.

[2] J. Moskovitz, E. Flescher, B.S. Berlett, J. Azare, J.M. Poston, E.R. Stadtman, Proc. Natl. Acad. Sci. USA 95 (1998) 14071-14075.

[3] D.B. Oien, J. Moskovitz, Curr. Top Dev. Biol. 80 (2008) 93-133.

[4] J. Moskovitz, Biochim. Biophys. Acta 1703 (2005) 213-219.

[5] C.C. Chao, Y.S. Ma, E.R. Stadtman, Proc. Natl. Acad. Sci. USA 94 (1997) 29692974.

[6] M.A. Ciorba, S.H. Heinemann, H. Weissbach, N. Brot, T. Hoshi, Proc. Natl. Acad. Sci. USA 94 (1997) 9932-9937.

[7] M. Mohri, P.S. Reinach, A. Kanayama, M. Shimizu, J. Moskovitz, T. Hisatsune, Y. Miyamoto, Invest. Ophthalmol. Vis. Sci. 43 (2002) 3190-3195.

[8] R.G. Midwinter, F.C. Cheah, J. Moskovitz, M.C. Vissers, C.C. Winterbourn, Biochem. J. 396 (2006) 71-78.

[9] Y. Xiong, B. Chen, H.S. Smallwood, R.J. Urbauer, L.M. Markille, N. Galeva, T.D. Williams, T.C. Squier, Biochemistry 45 (2006) 14642-14654.

[10] J.R. Erickson, M.L. Joiner, X. Guan, W. Kutschke, J. Yang, C.V. Oddis, R.K. Bartlett J.S. Lowe, S.E. O'Donnell, N. Aykin-Burns, M.C. Zimmerman, K. Zimmerman, A.J Ham, R.M. Weiss, D.R. Spitz, M.A. Shea, R.J. Colbran, P.J. Mohler, M.E. Anderson, Cell 133 (2008) 462-474.

[11] S. Swarup, M.C. Timmermans, S. Chaudhuri, J. Messing, Plant J. 8 (1995) 359368.

[12] R. Gonzalez-Iglesias, M.A. Pajares, C. Ocal, J.C. Espinosa, B. Oesch, M. Gasset, J. Mol. Biol. 319 (2002) 527-540.

[13] S.B. Prusiner, W.J. Hadlow, D.E. Garfin, S.P. Cochran, J.R. Baringer, R.E. Race, C.M. Eklund, Biochemistry 17 (23) (1978) 4993-4999 ( ${ }^{*}$ LHM: This journal is received by the Medical Library, Ein Kerem *LHC: 1962 - SHELF 4942.)

[14] B. Caughey, G.J. Raymond, S.A. Priola, D.A. Kocisko, R.E. Race, R.A. Bessen, P.T. Lansbury Jr., B. Chesebro, Mol. Biotechnol. 13 (1999) 45-55.

[15] T. Canello, R. Engelstein, O. Moshel, K. Xanthopoulos, M.E. Juanes, J. Langeveld, T. Sklaviadis, M. Gasset, R. Gabizon, Biochemistry 47 (2008) 8866-8873.

[16] N. Stahl, M.A. Baldwin, D.B. Teplow, L. Hood, B.W. Gibson, A.L. Burlingame, S.B. Prusiner, Biochemistry 32 (1993) 1991-2002.

[17] D.R. Brown, Folia Neuropathol. 43 (2005) 229-243.

[18] R.C. Nadal, S.R. Abdelraheim, M.W. Brazier, S.E. Rigby, D.R. Brown, J.H. Viles, Free Radic. Biol. Med. 42 (2007) 79-89.

[19] L. Redecke, M. von Bergen, J. Clos, P.V. Konarev, D.I. Svergun, U.E. Fittschen, J.A. Broekaert, O. Bruns, D. Georgieva, E. Mandelkow, N. Genov, C. Betzel, J. Struct. Biol. 157 (2007) 308-320.

[20] J.R. Requena, M.N. Dimitrova, G. Legname, S. Teijeira, S.B. Prusiner, R.L. Levine, Arch. Biochem. Biophys. 432 (2004) 188-195.

[21] D.T. Le, X. Liang, D.E. Fomenko, A.S. Raza, C.K. Chong, B.A. Carlson, D.L. Hatfield, V.N. Gladyshev, Biochemistry (2008), in press.

[22] M. Bruschi, A. Petretto, G. Candiano, L. Musante, E. Movilli, L. Santucci, A Urbani, R. Gusmano, E. Verrina, G. Cancarini, F. Scolari, G.M. Ghiggeri, J. Chromatogr. B Analyt. Technol. Biomed. Life Sci. 864 (2008) 29-37.

[23] C. Chumsae, G. Gaza-Bulseco, J. Sun, H. Liu, J. Chromatogr. B Analyt. Technol. Biomed. Life Sci. 850 (2007) 285-294. 